\title{
un nouvel outil pour la biologie et la médecine
}

\author{
Hervé RIGNEAULT \\ Aix Marseille Univ, \\ École Centrale de Marseille, CNRS, \\ Institut Fresnel UMR 7249, \\ 13397 Marseille, France \\ herve.rigneault@fresnel.fr
}

Les biomolécules peuvent être visualisées directement en microscopie optique en mesurant leurs modes vibratoires, et ceci dans des environnements complexes comme les cellules et les tissus.Cependant le passage des mesures spectroscopiques en cuvette à l'imagerie des systèmes vivants a nécessité des efforts tout particuliers qui ont aujourd'hui atteint une maturité capable d'apporter des solutions novatrices en biologie et en médecine. Cet article présente les grandes lignes des recherches et réalisations en imagerie spectroscopique vibrationnelle en partant des concepts de base pour allervers les applications.

cependant ses apports sont limités pour les applications in vivo du fait de la forte absorption de l'eau dans le proche et lointain IR. Ce problème peut être résolu en utilisant la diffusion Raman qui permet d'obtenir une signature des modes vibratoires moléculaires dans le domaine du visible où l'absorption par l'eau est faible (figure 1b). Découverte en 1928 par Chandrashekhara Venkata Râman [1] et indépendamment par Grigory Landsderg et Leonid Mandelstam [2] la diffusion Raman est un phénomène inélastique où une lumière incidente de pulsation $\omega_{1}$ est diffusée en une radiation $\omega_{1}-\Omega_{R}$, où $\Omega_{R}$ est la pulsation du mode vibratoire moléculaire (considéré ici comme unique). Une analyse spectroscopique de la lumière diffusée permet de remonter à $\Omega_{\mathrm{R}}$. Bien que permettant en principe de connaître les modes vibratoires de n'importe quelle molécule, et ceci dans un milieu aqueux, la diffusion Raman restera cependant peu utilisée jusque dans les années 60 (date de la découverte de l'effet laser) du fait de la faible section efficace Raman (1 photon sur 1 million de photons incidents est diffusé) et des signaux parasites (comme la fluorescence) qui viennent perturber l'analyse des spectres obtenus. Découverte peu de temps après l'invention du laser, la diffusion Raman cohérente [3] allait permettre d'obtenir un gain de sensibilité d'environ un million sur la diffusion Raman. Dans ce processus d'interaction lumière matière, deux ondes incidentes (idéalement des impulsions ultra courtes pour bénéficier de puissances crêtes importantes - de l'ordre $\mathrm{du} \mathrm{GW} / \mathrm{cm}^{2}$ ) de pulsation $\omega_{1}$ et $\omega_{1}-\Omega_{R}$ vont échanger leur énergie avec le mode vibratoire moléculaire. Quatre processus se réalisent alors qui conduisent soit à la création de nouvelles pulsations (effets CARS et CSRS - pour coherent anti-Stokes Raman scattering et coherent Stokes Raman scattering), soit à l'échange d'énergie entre les ondes incidentes (effets SRG et SRL - pour stimulated Raman gain et stimulated Raman loss) (figure 1c). Ces 2 processus, tous concomitants, sont les résultats de mélanges à quatre ondes résonants, c'est-à-dire impliquant le mode vibratoire $\Omega_{\mathrm{R}}$. Ils sont de plus cohérents et entretiennent une relation de phase précise avec les champs incidents à $\omega_{1}$ 


\section{L'OPTIQUE EST NOTRE AVENIR $^{\text {TM }}$}

et $\omega_{1}-\Omega_{\mathrm{R}}$. Ainsi les modes vibratoires, de toutes les molécules présentes dans la zone d'interaction lumière matière, vont vibrer de concert et les processus CARS, CSRS, SRL et SRG vont bénéficier de l'addition cohérente des ondes diffusées augmentant ainsi d'un facteur $10^{6}$ la sensibilité du Raman cohérent par rapport au Raman spontané. Tout d'abord exploitée à des fins spectroscopiques, la diffusion Raman cohérente allait bouleverser le domaine de l'imagerie moléculaire avec la construction du premier microscope CARS en 1982 par Duncan et al. [4] et grandement améliorée par Zumbusch et al. en 1999 grâce à l'utilisation de lasers à solides [5]. Ces travaux allaient lancer toute une communauté sur la voie de l'imagerie moléculaire sans marquage et subir une cure de jouvence en 2008 avec la réalisation des premiers microscopes SRS (stimulated Raman scattering) [6,7]. Aujourd'hui, la microscopie Raman cohérente s'affiche comme une technologie d'avenir incontournable dans les domaines de l'imagerie fonctionnelle des cellules et des tissus, du suivi de la pénétration d'actifs moléculaires dans les tissus et s'invite dans le monde du diagnostic in vivo et en temps réel du cancer [8].

\section{La mise en ouvre technologique}

La mise en œuvre d'une expérience de Raman cohérent nécessite d'avoir à disposition deux lasers impulsionnels de pulsations $\omega_{1}$ et $\omega_{1}-\Omega_{R}$ et présentant un taux de répétition précisément identique. Ceci afin de pouvoir assurer un recouvrement temporel des impulsions (à $\omega_{1}$ et $\omega_{1}-\Omega_{\mathrm{R}}$ ) au niveau de l'échantillon. Ce recouvrement est nécessaire pour activer les mélanges d'ondes générant les signaux CARS et SRS. Dans la terminologie consacrée du Raman cohérent on appelle 'pompe' l'impulsion incidente de plus haute énergie $\omega_{\mathrm{P}}=\omega_{1}$ et 'Stokes' celle de plus basse énergie. Quand $\omega_{S}=\omega_{1}-\Omega_{R}$, c'est-à-dire $\omega_{\mathrm{P}}-\omega_{\mathrm{S}}=\Omega_{\mathrm{R}}$, le mélange d'onde entre les impulsions pompe et Stokes est résonant avec un mode vibratoire moléculaire $\Omega_{\mathrm{R}}$, et les processus
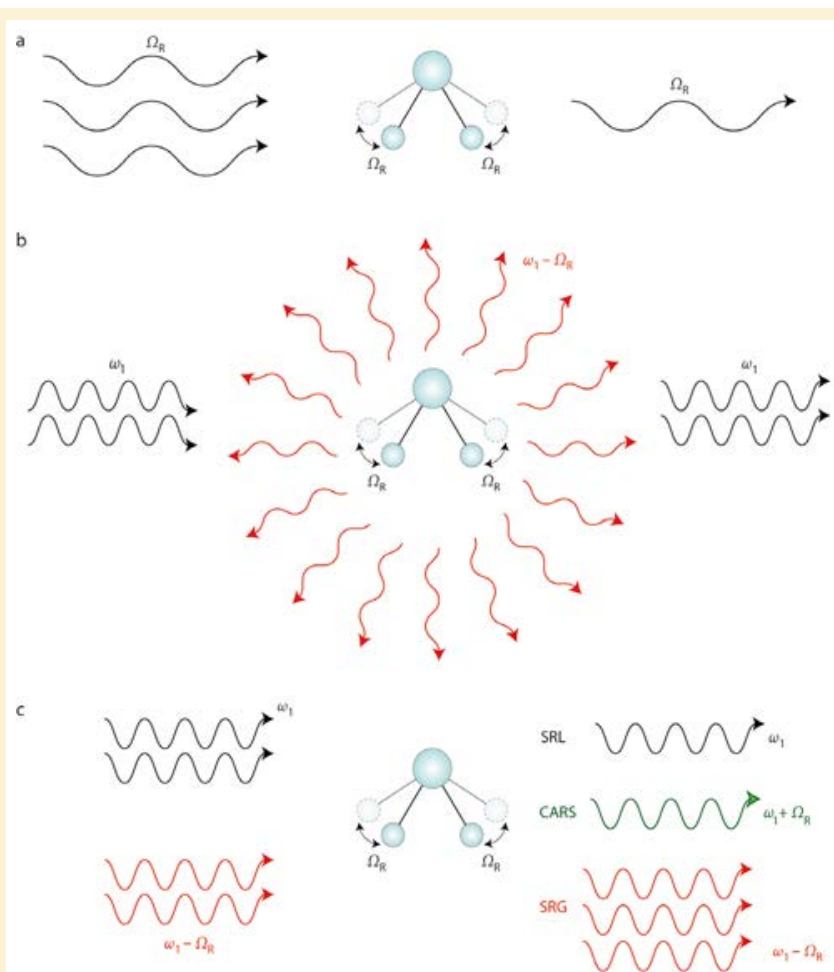
l'onde à $\omega_{1}$ (effet SRL - stimulated Raman loss), (ii) une amplification de l'onde à $\omega_{1}-\Omega_{R}$ (effet SRG - stimulated Raman gain) et (iii) la création d'une nouvelle onde à la fréquence $\omega_{1}+\Omega_{R}$ (effet CARS - coherent anti-Stokes Raman scattering). Une dernière onde, non représentée, est également créée à la fréquence $\omega_{1}-2 \Omega_{R}$ (effet CSRS - coherent Stokes Raman scattering) mais elle est généralement non utilisée en imagerie.
Figure 1. Les

processus

d'interaction entre

les modes vibratoires moléculaires et la lumière. (a) Cas de l'absorption infrarouge où une radiation incidente de pulsation $\Omega_{R}$ est absorbée par un mode vibratoire à $\Omega_{R}$. (b) Cas de la diffusion Raman où une radiation incidente de pulsation $\omega_{1}$ est diffusée inélastiquement avec un changement de fréquence $\omega_{1}-\Omega_{R}$. (c) Cas de la diffusion Raman cohérente où deux ondes de pulsation $\omega_{1}$ et $\omega_{1}-\Omega_{R}$ interagissent avec un mode vibratoire moléculaire pour donner naissance à (i) une déplétion de .

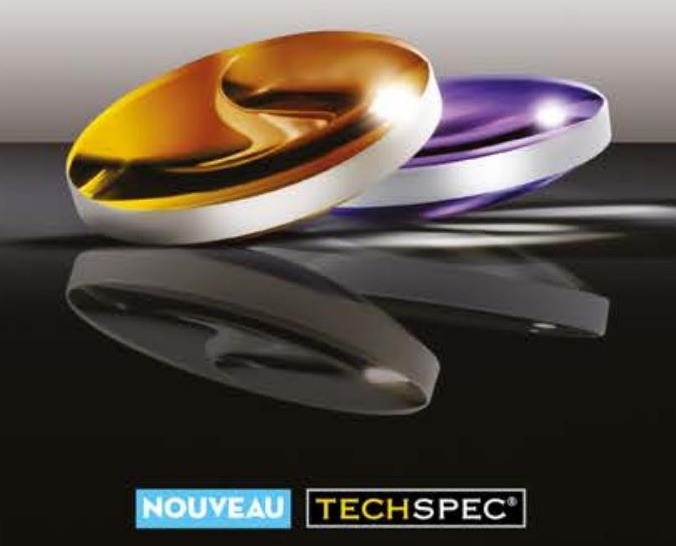

\section{Lentilles Asphériques de Qualité Laser $\lambda / 40$}

- Tolérance de surface asphérique (RMS) de $\lambda / 40$

- Rapport de Strehl garanti >0,8

- Traitements raie laser en $\mathrm{V}$ avec une réflexion $<0,25 \%$

Les Lentilles Asphériques de Qualité Laser TECHSPEC ${ }^{\oplus}$ sont fabriquées par MRF de précision. La surface ultralisse offre une performance limitée par la diffraction à la longueur d'onde de conception. Avec un substrat de silice fondue et une qualité de surface de $10-5$, ces lentilles sont conçues pour les applications les plus exigeantes.

Pour en savoir plus sur

nos capacités de production

www.edmundmanufacturing.fr

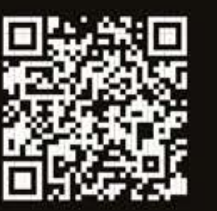

$\triangleright$ Rendez-nous visite à

LASER IPHOTONICS

Munich, 24 - 27 Juin 2019 Hall B1, Stand 511

+33(0) 820207555

sales@edmundoptics.fr 


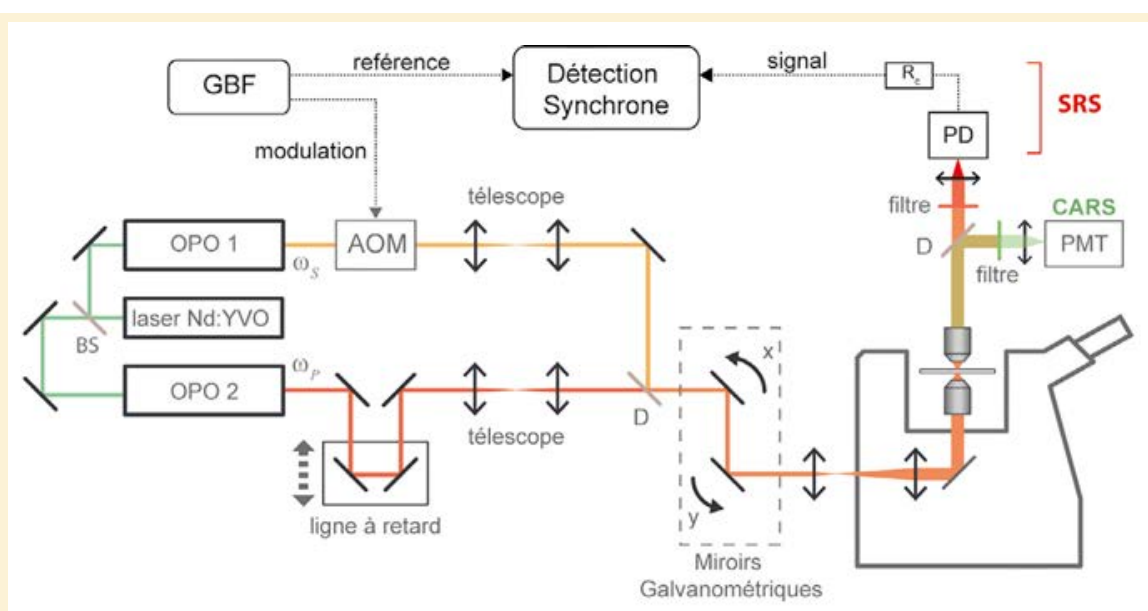

Figure 2. Un microscope Raman cohérent utilise des oscillateurs paramétriques optiques (OPO) qui délivrent des trains d'impulsions de fréquence précisément identique. Les impulsions pompe et Stokes, synchronisées temporellement, sont focalisées sur l'échantillon où elles activent les contrastes CARS et SRS. Le signal CARS est détecté à l'aide d'un photomultiplicateur (PMT) alors que le signal SRS utilise une photodiode (PD) et un amplificateur à détection synchrone. GBF : générateur basse fréquence, Rc : résistance de charge, $\mathrm{AOM}$ : modulateur acousto-optique, $\mathrm{D}$ : miroir dichroïque de recombinaison.

CARS et SRS présentent une sélectivité chimique. Si $\omega_{\mathrm{P}}-\omega_{\mathrm{S}} \neq \Omega_{\mathrm{R}}$ alors les processus CARS et SRS sont faibles ou nuls car non résonants avec les modes vibratoires, ils ne présentent alors pas de sélectivité chimique. Idéalement l'un au moins des lasers doit être accordable en longueur d'onde pour pouvoir faire varier la différence de fréquence $\omega_{\mathrm{P}}-\omega_{\mathrm{S}}$ et ainsi pouvoir adresser un mode vibratoire moléculaire quelconque. On note dans la littérature plusieurs types de mise en oeuvre de la microscopie Raman cohérente dont la plus courante est celle indiquée sur la Figure 2. Le montage utilise des oscillateurs paramétriques optiques $(\mathrm{OPO})$ qui ont la propriété d'être accordables en fréquence (et donc en pulsation $\omega$ ). Dans la pratique un des OPO garde une longueur d'onde fixe (OPO1) et on fait varier la longueur d'onde de $\mathrm{OPO} 2$ (dans le proche IR) de telle sorte que $\omega_{\mathrm{P}}-\omega_{\mathrm{S}}=\Omega_{\mathrm{R}}$ (où $\left.\omega=\frac{2 \pi \mathrm{c}}{\lambda}\right)$. On exprime alors en $\mathrm{cm}^{-1}$ l'écart en fréquence des deux impulsions pompe et Stokes et le système peut ainsi balayer tout le spectre vibrationnel des liaisons chimiques existantes situées entre $400 \mathrm{~cm}^{-1}$ et $4000 \mathrm{~cm}^{-1}$.

Dans la pratique on préfere utiliser des impulsions dont la durée est de l'ordre de quelques ps $\left(10^{-12}\right.$ s), ceci afin d'assurer une résolution spectrale de quelques $10 \mathrm{~cm}^{-1}$ dans photomultiplicateur. Le signal SRS nécessite une instrumentation plus lourde ; il s'agit de moduler un des faisceaux et d'observer le transfert de modulation lié à l'effet SRS sur l'autre faisceau (cas de OPO2 $\omega_{\mathrm{P}}$ sur la figure 2). En pratique les signaux CARS et SRS sont acquis conjointement.

\section{Quelques applications de l'imagerie Raman cohérente}

La microscopie Raman cohérente permet d'imager les modes vibratoires moléculaires sans avoir à préparer l'échantillon d'aucune façon. Activés seulement au point de focalisation d'un objectif de microscope que l'on balaye dans l'échantillon, les contrastes CARS et SRS produisent des images tridimensionnelles avec une résolution en dessous du micromètre. Les liaisons carbone/hydrogène $\mathrm{CH}_{2}$ et $\mathrm{CH}_{3}$ donnent d'importants signaux en Raman cohérent à cause de la forte section efficace Raman de ces modes vibratoires mais aussi parce que ces liaisons sont présentes en grand nombre dans les protéines et les lipides. L'organisation des couches lipidiques joue en particulier un rôle très important dans le caractère fonctionnel de la myéline. Cette substance grasse est trouvée dans certaines cellules du système nerveux ainsi que dans le système nerveux périphérique (les nerfs). La myéline entoure les fibres nerveuses et sert à les isoler et à les protéger, comme le fait le plastique autour des fils électriques. Dans le cas de certaines maladies comme la sclérose en plaque, les gaines de myéline 
subissent des affections majeures qui conduisent à leur déstructuration. La figure 3 montre des images CARS des gaines de myélines (en rouge) au cours de la progression d'une sclérose en plaque chez un modèle murin (encéphalomyélite allergique expérimentale - EAE). Dans le cas de souris saines, on observe comment la gaine de myéline entoure tout l'axone (ici marqué avec la protéine fluorescente CFP) : lors de l'évolution de la maladie, la myéline se déstructure en amas globulaires qui finissent par envahir tout l'espace inter-axone, produisant alors des déficiences motrices majeures chez les individus atteints [9]. Les liaisons carbone/hydrogène ne sont pas les seules qui peuvent être imagées à l'aide du Raman cohérent. En principe toutes les liaisons chimiques qui signent en Raman peuvent être suivies et imagées. Dans la pratique cette spécificité moléculaire est cependant limitée car les cellules et tissus sont constitués eux (a)

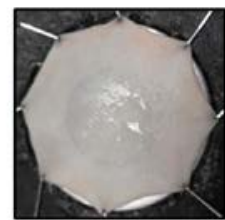

Peau abdominale humaine

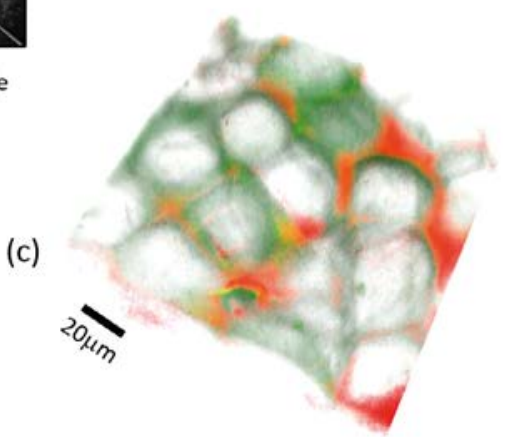

Molécule active LR2412 (CARS)

Kératinocyte (TPEF)

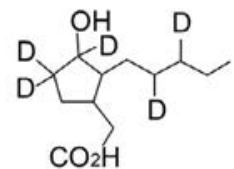

Molécule active deutérée LR2412

Figure 4. Étude de la pénétration d'actifs moléculaires dans la peau par microscopie

Raman cohérente. (a) Échantillon de peau humaine abdominale mis au contact (b) d'une molécule deutérée active en cosmétique. (c) Répartition de l'actif (CARS - rouge) au sein de l'épiderme (auto-fluorescence à deux photons - vert).

aussi de protéines et de lipides dont les signatures Raman sont présentes; le monde du vivant est construit avec les mêmes liaisons chimiques ! Afin de retrouver une spécificité moléculaire il est intéressant d'utiliser un marqueur isotopique comme par exemple le deutérium. Cet isotope de l'hydrogène n'altère pas la structure et la fonctionnalité des bio-molécules. La liaison carbone / deutérium est particulièrement intéressante car elle présente
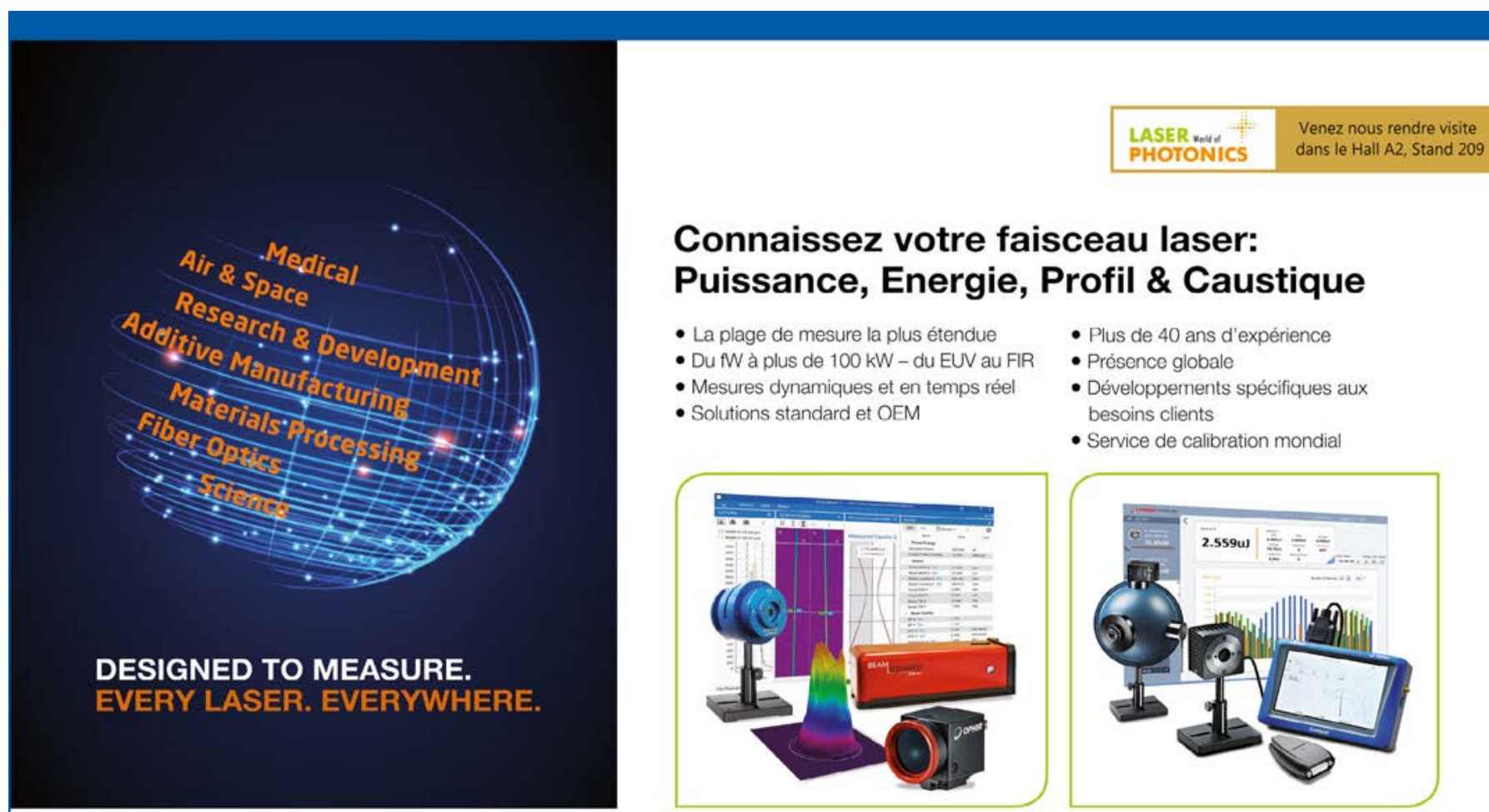

Connaissez votre faisceau laser: Puissance, Energie, Profil \& Caustique

- Du fW à plus de 100 kW - du EUV au FIR

- Plus de 40 ans d'expérience

- Présence globale

- Développements spécifiques aux

besoins clients

- Service de calibration mondial

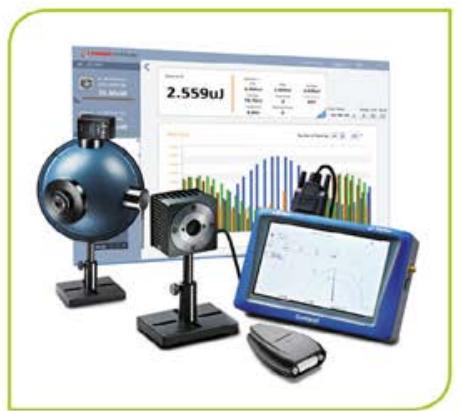

www.ophiropt.com/photonics 

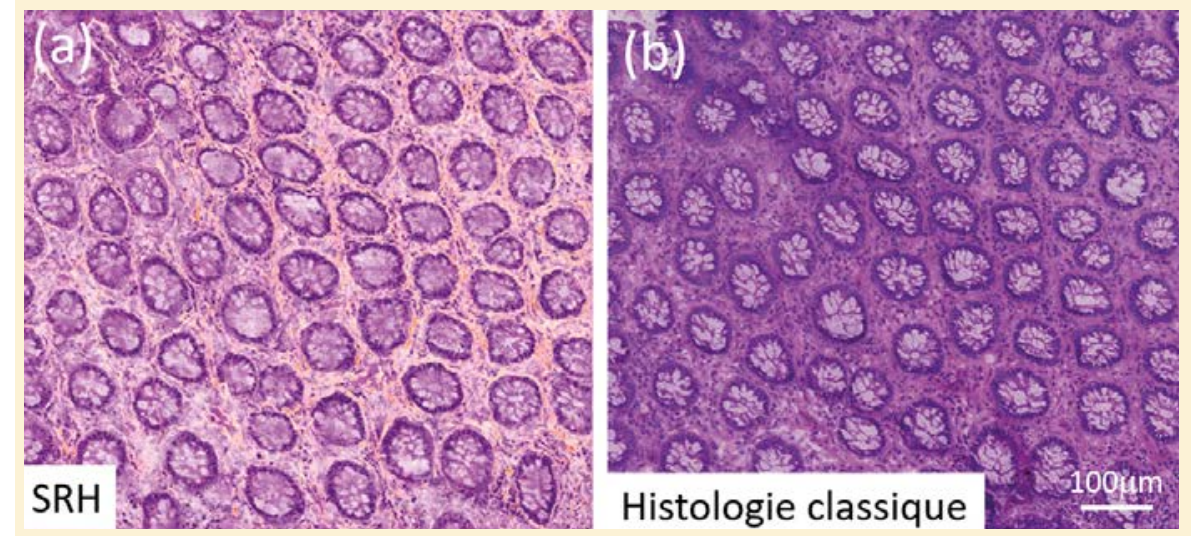

Figure 5. Comparaison des images SRH (stimulated Raman histology) (a) et histologique (marquage éosine et hématoxyline) (b) sur un échantillon de colon sain humain.

une signature Raman à $2100 \mathrm{~cm}^{-1}$, un domaine spectral quasiment vierge de modes vibratoires pour les molécules endogènes que l'on trouve dans les systèmes vivants. Nous avons utilisé récemment cette stratégie pour imager des composés moléculaires actifs en pharmacologie et en cosmétique pénétrant dans la peau humaine [10]. La figure $4 a$ présente des échantillons de peau abdominale humaine qui sont utilisés et mis en contact avec les molécules actives dont on veut suivre la pénétration transcutanée. En utilisant la signature $\mathrm{C}-\mathrm{D}$, il est alors possible d'imager la répartition d'un composé actif (figure $4 b$ - cas de la molécule LR2412 utilisée dans la crème anti-âge Visionnaire commercialisée par Lancôme) dans l'épiderme. La figure $4 c$ précise le mode de pénétration de cette molécule active (en rouge) qui se localise entre les cornéocytes (cellules de la surface de la peau) imagées ici par leur signal d'autofluorescence à deux photons (en vert).

\section{Perspectives}

Déjà largement utilisé pour étendre le champ des connaissances en biologie aussi bien au niveau cellulaire que tissulaire, le Raman cohérent devient désormais un outil d'imagerie incontournable grâce à de nouvelles perspectives [8]. C'est tout d'abord l'utilisation des marquages alkyne (carbone triple liaison hydrogène, $-\mathrm{C} \equiv \mathrm{C}-$ ) qui permet de suivre les synthèses d'ADN et d'ARN ainsi que le métabolisme de molécules médicamenteuses directement dans les cellules. Le marquage par des groupes alkynes a cette propriété d'être 'bio-orthogonal', c'est-à-dire de ne pas interférer avec le métabolisme, il est alors possible de suivre comment une structure moléculaire est métabolisée par la machinerie cellulaire [11]. Dans le domaine biomédical, des études récentes démontrent la possibilité d'utiliser les images SRS acquises sur les bandes $\mathrm{CH}_{2}\left(2850 \mathrm{~cm}^{-1}\right)$ et $\mathrm{CH}_{3}\left(2950 \mathrm{~cm}^{-1}\right)$ pour détecter la progression de tumeurs dans les tissus. Cette approche appelée histologie Raman stimulée (SRH - stimulated Raman bistology)

\section{POUR EN SAVOIR PLUS} $350(1982)$ Phys. Rev. Lett. 82, 4014 (1999) Raman scattering microscopy," Science 322, 1857 (2008) py," New J. Phys. 11, 033026 (2009) permet d'obtenir des images quasiment identiques à celles obtenues à l'hôpital en histologie classique mais dans des temps cinquante fois plus courts (quelques dizaines de minutes pour le SRH contre 10-24 h pour l'histologie classique) $[12,13]$. La figure 5 présente la comparaison entre une image SRH et celle obtenue en histologie classique et démontre l'intérêt du SRH dans un contexte intra-opératoire pour détecter rapidement des tissus cancéreux. Des perspectives s'ouvrent également du côté instrumental où des efforts sont menés pour réaliser une imagerie Raman cohérente en endoscopie. Pour cela de nouvelles fibres optiques comportant un coeur creux sont réalisées ; elles ont la propriété de transporter des impulsions ultra-courtes sans distorsions spectrales et temporelles, nécessaires à l'activation des contrastes CARS et SRS [14]. Depuis sa première mise en oeuvre en 1982, l'imagerie Raman cohérente n'a cessé de puiser sa force dans les avancées technologiques en optoélectronique et dans l'ingénierie moléculaire, tout cela devrait continuer, et il y a fort à parier que ces développements vont mener l'imagerie CARS et SRS vers les laboratoires de biologie, l'hôpital et les applications industrielles.

[1] C. V. Raman, K. S. Krishnan, "A new radiation, " Indian Journal of Physics 2, 387 (1928)

[2] G. S. Landsberg, L. I. Mandelstam, "New phenomenon in scattering of light (preliminary report)," Journal of the Russian Physico-Chemical Society, Physics Section 5, 335 (1928)

[3] N. Bloembergen, "The stimulated Raman effect, " American Journal of Physics 35, 989 (1967)

[4] M. D. Duncan et al., "Scanning coherent anti-Stokes Raman microscope," Optics Letters. 7,

[5] A. Zumbusch et al., "Vibrationnal microscopy using coherent anti-Stokes Raman scattering,"

[6] C. W. Freudiger et al.,"Label-free biomedical imaging with high sensitivity by stimulated

[7] P. Nandakumar et al., "Vibrational imaging based on stimulated Raman scattering microsco-

[8] J. X. Cheng, X. S. Xie, "Vibrational spectroscopic imaging of living systems: An emerging platform for biology and medicine, "Science 350, 1054 (2015)

[9] P. Gasecka et al., "Lipid order degradation in autoimmune demyelination probed by polarized coherent Raman microscopy, "Biophysical Journal 113, 1520 (2017)

[10] X. Chen et al., "Quantitative 3D molecular cutaneous absorption in human skin using label free nonlinear microscopy," Journal of Controlled Release 200, 78 (2015) 